10. Swedish Museum of Natural History, Bird Ringing Centre. Annual Reports of Swedish Bird Ringing 1960-98 (ISSN 0282-390X).

11. Thorup, K. \& Rabøl, J. J. Avian Biol. 32, 111-119 (2001).

12. International Association of Geomagnetism and Aeronomy. Geophys. J. Int. 141, 259-262 (2000).

13. Lohmann, K. \& Lohmann, C. M. F. J. Exp. Biol. 194, 23-32 (1994).

COMMUNICATIONS ARISING

Tumour suppressors

\section{Effect of DNA damage on a BRCA1 complex}

- he tumour-suppressor protein BRCA1 mediates its biological functions by

interacting with cellular factors ${ }^{1,2}$ such as the CtIP polypeptide ${ }^{3,4}$, a substrate for the ATM (for 'ataxia telangiectasia mutated') protein kinase ${ }^{5}$. Li et al. ${ }^{6}$ report that the BRCA1-CtIP interaction is disrupted by ionizing radiation and by other genotoxic stresses that induce phosphorylation of CtIP by ATM kinase, and that this dissociation of the BRCA1-CtIP complex in turn modulates the transcription of DNA-damage-response genes $^{6}$. We have shown that the BRCA1binding domain of CtIP (amino-acid residues 133-369) is distal to the sites that are phosphorylated by ATM kinase (residues S664 and S745) ${ }^{7}$. We now show that the BRCA1-CtIP complex is stable in irradiated cells, and that the phosphorylated isoforms of CtIP that are induced by ionizing radiation still interact in vivo with BRCA1. We conclude that disruption of the BRCA1-CtIP complex cannot account for induction of DNA-damage-response genes in the way proposed by Li et al. ${ }^{6}$.

To investigate the effect of genotoxic stress on CtIP, we treated human T24 carcinoma cells with ionizing radiation or ultraviolet light, and immunoblotted the cell lysates with a CtIP-specific monoclonal antibody ${ }^{7}$. As expected, ionizing radiation induced the formation of the phosphorylated CtIP isoforms ${ }^{6}$ (Fig. 1a, top, lane 3). These species migrate more slowly than CtIP polypeptides from untreated cells (lane 1) and are converted to a faster-migrating form after incubation with $\lambda$-phosphatase (lane 4). As expected, BRCA1 was also hyperphosphorylated in cells exposed to ionizing radiation (Fig. 1a, bottom) $)^{8,9}$.

To determine the effect of ionizing radiation on the BRCA1-CtIP complex, we immunoprecipitated T24-cell lysates with a BRCA1-specific polyclonal antiserum, and monitored each precipitate for CtIP by immunoblotting with a CtIP-specific monoclonal antibody. As expected, CtIP was detected in BRCA1 immunoprecipitates from lysates of untreated and ultravioletirradiated cells (Fig. 1b, top , lanes 5, 9), which is consistent with our previous findings ${ }^{7}$ but not with those of Li et al. ${ }^{10}$.

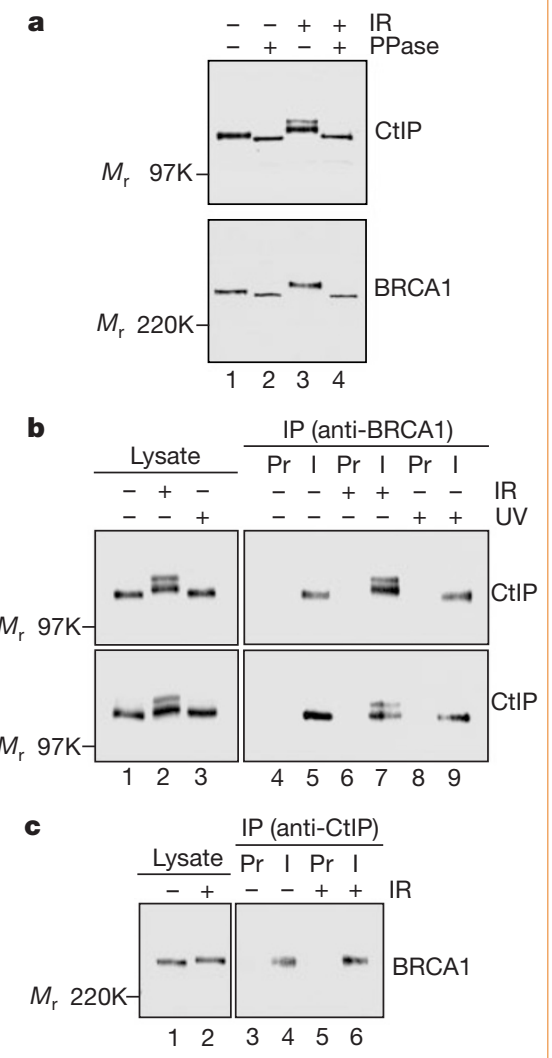

Figure 1 Association of BRCA1 and CtIP in irradiated cells. a, Induction of CtIP phosphorylation. Lysates from T24 carcinoma cells (lanes 1, 2, untreated; lanes 3, 4, treated with 40 Gy ionizing radiation $(\mathrm{IR})$ were immunoblotted with CtIP-specific (top) or BRCA1specific (bottom) monoclonal antibodies ${ }^{7}$; the indicated lysates were pretreated with $\lambda$-phosphatase (PPase). $\mathbf{b}$, Co-immunoprecipitation of CtIP with BRCA1. T24 cells (top) and GM000637H fibroblasts (bottom) were irradiated with 40 Gy IR or $10 \mathrm{~J} \mathrm{~m}^{-2}$ ultraviolet (UV) light, and lysates were immunoblotted for CtIP (lanes 1-3). Alternatively, lysates were immunoprecipitated with BRCA1-specific antiserum $^{7}(\mathrm{I})$ or pre-immune serum (Pr) and then immunoblotted for CtIP (lanes 4-9). c, Co-immunoprecipitation of BRCA1 with CtIP. Lysates of HBL100 epithelial cells were either immunoblotted for BRCA1 (lanes 1, 2) or immunoprecipitated with CtIP-specific 210 antiserum $^{7}$ and then immunoblotted for BRCA1 (lanes 3-6).

Moreover, we recovered all CtIP species, including the hyperphosphorylated forms, in BRCA1 immunoprecipitates prepared from cells exposed to ionizing radiation (Fig. 1b, lane 7). The same results were obtained in all cell lines tested, including the two lines examined by $\mathrm{Li}$ et al. ${ }^{6}$ : T24 carcinoma cells (Fig. 1b, top) and SV40-transformed GM000637H fibroblasts (Fig. 1b, bottom).

The stability of the CtIP-BRCA1 complex was also evident in reciprocal coimmunoprecipitation experiments, in which comparable amounts of BRCA1 polypeptides were present in CtIP immunoprecipitates from both untreated cells and cells exposed to ionizing radiation (Fig. 1c, lanes 4, 6). Our results indicate that, contrary to the findings of Li et al., the CtIP-BRCA1 complex is stable to genotoxic stress such as ultraviolet or ionizing radiation.

Foon Wu-Baer, Richard Baer
Institute of Cancer Genetics and Department of Pathology, Columbia University College of Physicians and Surgeons, 1150 St Nicholas Avenue, New York, New York 10032, USA e-mail: rb670@columbia.edu

1. Scully, R. \& Livingston, D. M. Nature 408, 429-432 (2000).

2. Deng, C.-X. \& Brodie, S. G. BioEssays 22, 728-737 (2000).

3. Yu, X., Wu, L. C., Bowcock, A. M., Aronheim, A. \& Baer, R. J. Biol. Chem. 273, 25388-25392 (1998).

4. Wong, A. K. et al. Oncogene 17, 2279-2285 (1998).

5. Kim, S. T., Lim, D. S., Canman, C. E. \& Kastan, M. B. J. Biol. Chem. 274, 37538-37543 (1999).

6. Li, S. et al. Nature 406, 210-215 (2000).

7. Yu, X. \& Baer, R. J. Biol. Chem. 275, 18541-18549 (2000).

8. Scully, R. et al. Cell 90, 425-435 (1997).

9. Thomas, J. E., Smith, M., Tonkinson, J. L., Rubinfeld, B. \& Polakis, P. Cell Growth Differ. 8, 801-809 (1997). 10. Li, S. et al. J. Biol. Chem. 274, 11334-11338 (1999).

Li et al. reply - Wu-Baer and Baer confirm our original observation that CtIP is phosphorylated in an ATM-dependent manner in response to $\gamma$-radiation. We have shown that phosphorylation by ATM kinase of CtIP at serine residues 664 and 745 is required to liberate DNA-damage-response genes such as GADD45 from repression. This is consistent with our more recent finding that overexpression in mammalian cells of a phosphorylated CtIP mutant with a double alanine substitution at serines 664 and 745 disrupts the radiation-induced cell-cycle checkpoint between $\mathrm{G}_{2}$ and $\mathrm{M}$ phases. The functional consequence of radiation-induced, ATM-dependent phosphorylation of CtIP is therefore clear.

With respect to the mechanism that underlies this process, we proposed that phosphorylation of CtIP leads to its dissociation from BRCA1, freeing BRCA1 to participate in the activation of DNAdamage-response genes. We do, however, appreciate the potential for experimental artefact in using only soluble co-immunoprecipitation to detect protein-protein interactions, particularly when different antibodies and cell lines are used. (We used human colon cancer cell line HCT 116 and human fibroblasts GM09607A and GM00637G, whereas Wu-Baer and Baer used human bladder carcinoma cell line T24 and fibroblast GM000637H.)

Wu-Baer and Baer report reciprocal coimmunoprecipitation of BRCA1 and CtIP using two different cell lines instead of the same cells. Their observations are inconsistent with ours where the interaction status of BRCA1 and CtIP after $\gamma$-irradiation is concerned. This discrepancy should eventually be resolved by systematic investigation using an alternative and complementary method.

Shang Li, Nicholas S.Y. Ting, Lei Zheng, Phang-Lang Chen, Wen-Hwa Lee

Department of Molecular Medicine, University of Texas Health Science Center at San Antonio, Institute of Biotechnology, San Antonio,

Texas 78245-3207, USA

e-mail: leew@uthscsa.edu 